\title{
Originales
}

\section{¿Presenta desgaste profesional el personal de urgencias extrahospitalarias? Resultados Encuestas de Malasch}

\author{
Are out-of-Hospital Emergency Medical Services Suffering from Professional \\ Burn-Out? Maslach Burnout Inventory Results
}

\section{Tania Arenal-Gota', Juan Luis Viana-Gárriz² ${ }^{2}$ Tomás Belzunegui-Otano³}

1. Complejo Hospitalario de Navarra. Dirección de Formación CPF emergencias. España.

2. Dirección de Operaciones de CPF emergencias. España.

3. Subdirección de urgencias y hospitalización del Complejo Hospitalario de Navarra. Navarra. España.

Recibido: 02-11-2018

Aceptado: 25-02-2019

\section{Correspondencia}

Tania Arenal Gota

C/ Santos Ochandategui 32, $3^{\circ} \mathrm{B}$

31012. Pamplona. Navarra. España

Teléfono: 649320137

Correo electrónico: taniaarena|@hotmail.com

\section{Resumen}

Objetivo: Determinar la prevalencia del Burnout en el personal de urgencias extrahospitalarias.

Material y métodos: Estudio observacional, descriptivo y transversal. Se les aplicó una ficha con unos datos profesionales y el cuestionario de Maslach de 22 items y se realizó un análisis estadístico descriptivo basado en obtener las frecuencias tanto absolutas como relativas. Además de la media y la desviación típica, se ha realizado un contraste de la hipótesis de Kruskal-Wallis y la de Wilcoxon. Para analizar la fiabilidad y validez de la escala se ha obtenido los coeficientes alfa de Cronbach para las 3 subescalas.

Resultados: Respecto al tiempo trabajado dentro de la categoría profesional los conductores que llevan menos de un año presentan mayor burnout. En relación al tipo de contrato, son los de contrato fijo. A peor estado de salud (regular o malo) mayor es el burnout. Las personas solteras presentan mayor cansancio emocional y no trabajar con los mismos compañeros aumenta también la presencia de este fenómeno.

El burnout no se presenta en los trabajadores con turno de 24 horas, sin embargo, se ve en los otros turnos. No hay diferencias estadísticas en la puntuación por el escaso tamaño de la muestra con jornada reducida.

Conclusiones: El interés de este estudio radica en conocer el grado de cansancio emocional que presentan los profesionales de urgencias extrahospitalarias y evidenciar la necesidad de tomar medidas preventivas. Los resultados de nuestro estudio están en la línea de los publicados.

Med Segur Trab (Internet). 2019;65(254):24-36

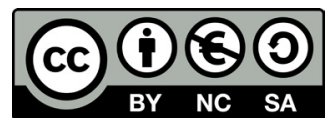

https://creativecommons.org/licenses/by-nc-sa/4.0/ 
Palabras clave: Servicios Médicos de Urgencia, Ambulancias, Estrés laboral, Trabajadores, Agotamiento profesional.

Abstract

Objective: To determine the prevalence of Burnout in out-of-hospital emergency medical personnel.

Material and methods: Observational, descriptive and transversal study. A professional data sheet and the Maslach Burnout Inventory of 22 items were applied. In order to get the absolute and relative frequencies a descriptive statistical analysis was performed. In addition to the mean and the standard deviation, a contrast to the Kruskal-Wallis hypothesis and the Wilcoxon hypothesis was shown. In order to analyze the reliability and validity of the scale, Cronbach's alpha coefficients were obtained for the 3 subscales.

Results: Regarding the time worked in the professional category, the drivers who have been driving for less than a year have higher levels of burnout. In relation to the type of contract are those of fixed-term contract. The worse the state of health (regular or bad) is, the greater the burnout. Single people have greater emotional exhaustion. The fact of not working with the same partners also increases this phenomenon. Burnout does not occur in workers with 24-hour shift, but it occurs in other shifts. Due to the small size of the short-time working sample, no statistical differences were found in the score.

Conclusions: The interest of this study lies in knowing the emotional exhaustion level from the out-ofhospital emergency professionals, as well as evidencing the need to take preventive measures. The results of our study are in line with those published.

Med Segur Trab (Internet). 2019;65(254):24-36

Keywords: Emergency Medical Services, Ambulances, Occupational Stress, Workers, Professional Burnout. 


\section{INTRODUCCIÓN}

La palabra Burnout traducida del inglés como "quemado" es utilizada en términos clínicos para hacer referencia al estado avanzado de desgaste profesional o síndrome de cansancio emocional (Gil-Montes, 2003).

El síndrome de Burnout es reconocido desde 1974 gracias a las publicaciones de Herbert Freudemberger, que fue el primero en hablar de burnout el comportamiento que presentó el personal de salud encargado de un programa de adolescentes delincuentes, ya que inicialmente se abordó desde los servicios sociales, lo definió como "el estado de fatiga o frustración que se produce por la dedicación a una causa, forma de vida o relación que no produce el resultado esperado"2.

El burnout se encuentra asociado a una gran cantidad de variables, principalmente, de tipo sociodemográficas, personales, de salud y laborales ${ }^{3}$. Existen factores endógenos y de personalidad que predisponen al burnout pero no son su origen.

Posteriormente en 1982, Cristina Maslach realizó el cuestionario compuesto por 22 items que pretende objetivar y valorar las 3 características básicas del síndrome: el agotamiento emocional, la despersonalización y el rendimiento laboral ${ }^{4}$.

El Burnout Ocupacional se identifica como un estado emocional alterado que, según Maslach y Jackson se puede evaluar a través de tres dimensiones; agotamiento emocional, cuando el trabajador se siente emocionalmente vacío, agotado por tener que lidiar con demandas que son abrumadoras; despersonalización, cuando el trabajador se vuelve insensible a los usuarios del servicio o del trabajo que se realiza, tratándolos o dirigiéndolos de manera cínica y deshumanizada y reduciendo el logro personal, cuando la persona experimenta fuertes sentimientos de incompetencia y fracaso ${ }^{5}$.

Cuando se produce Burnout los pacientes son vistos como meros "casos", lo cual se manifiesta en la respuesta fría e impersonal hacia ellos y puede ocasionar trastornos conductuales de aislamiento e insensibilidad en el profesional y el sentimiento de baja realización en el trabajo ${ }^{6}$. En un estudio realizado en Australia ${ }^{7}$ el $60 \%$ de los errores en la medicación están asociados al estrés del personal.

En la literatura que existe sobre burnout se expone como una problemática muy generalizada, que cada vez son más los profesionales afectados y son muchas las organizaciones laborales en las que resulta interesante estudiarlo. Se puede afirmar, que el desarrollo de una labor profesional en situaciones en las que existe gran implicación emocional hace más probable que se desarrolle el síndrome de Burnout (Jones, 2013).

Las consecuencias del burnout son múltiples como: el descenso de la calidad de los cuidados, aparición de problemas músculo esqueléticos, la depresión, la obesidad, el insomnio, el alcohol o tomar drogas de abuso $9,10,11,12$.

Dickinson C, et al 2016, afirmaron que son necesarias herramientas que ayuden a controlar el estrés de los profesionales sanitarios, las técnicas cognitivas son necesarias en el cuidado prehospitalario de niños.

Un estudio cualitativo realizado por Cushman et al, 2010 encuentra que el estrés y la ansiedad están relacionados directamente con la falta de experiencia que contribuye a errores.

En un estudio realizado en España entre personal de emergencias y personal de no emergencias no se observaron diferencias significativas entre sexos pero sí entre la organización del trabajo de las distintas empresas (Gonzalez, M. et al 2017), además llegaron a la conclusión de que los turnos de 24 horas les es favorable para conciliar mejor su vida familiar y laboral.

En el reciente proyecto financiado por la Unión Europea llevado a cabo por Matrix (2013), se estimó que el coste para Europa de la depresión relacionada con el trabajo puede ser de 617000 millones euros anuales ${ }^{13}$ Datos obtenidos en un estudio realizado 
en el Servicio de Asistencia Municipal de Urgencia y Rescate (SAMUR), accidentes de trabajo sin baja por agresiones $132(8,36 \%)$ y con baja $64(6,7 \%)^{14}$.

Respecto al género aparece un mayor burnout en varones que en mujeres ${ }^{15}$. En varias investigaciones se obtuvieron como resultados que los enfermeros presentaban niveles de burnout más altos que el resto de grupos profesionales estudiados, debido al turno, a la sobrecarga de trabajo y al contacto continuado con el paciente ${ }^{16,17}$.

El objetivo de este estudio fue determinar la prevalencia de Burnout o desgaste profesional en el personal de las urgencias extrahospitalarias e identificar los posibles factores que podrían estar asociados al síndrome de burnout.

\section{METODOLOGÍA}

\section{Diseño del estudio}

Estudio descriptivo, observacional y transversal. Mediante encuestas anónimas y autoadministradas realizadas mediante plataforma Survio en internet destinada a tal fin.

\section{Sujetos}

La muestra estaba formada por: 190 profesionales sanitarios de las urgencias extrahospitalarias residentes en España.

De los encuestados 12 (6,3\%) son médicos, 21 son enfermeras (11\%), 112 son Conductores Técnicos en Emergencias Sanitarias (58,6\%), 46 son Auxiliares Técnicos en Emergencias Sanitarias $(24,1 \%)$.

\section{Obtención de la muestra}

A la encuesta se le dio difusión y acceso a través de las redes sociales como Facebook y Twitter, y el blog de la principal autora del artículo, fue un método de máxima y rápida difusión, de fácil acceso a profesionales de las distintas Comunidades autónomas de este país.

El acceso a la encuesta estuvo abierto desde el 12 de Febrero de 2017 al 25 de Marzo de 2017 , un total de 42 días. En ella, se pedía como requisito ser personal de urgencias extrahospitalarias de España.

Los participantes fueron informados previamente del objetivo principal del estudio, en la nota aclaratoria al inicio de la encuesta se comunicaba que su participación era voluntaria y anónima y que los resultados obtenidos no iban a ser utilizados con fines ajenos a los de la investigación.

\section{Variables Estudiadas}

En esta investigación se ha trabajado con las siguientes variables todas ellas en relación con el cansancio emocional, la despersonalización y la realización personal.

- Puesto de trabajo

- Antigüedad en el centro

- Tipo de Contrato

- Duración de la jornada

- Turno

- Zona de trabajo

- Estado de salud

- Estado Civil

- Trabajar siempre con los mismos compañeros 


\section{Instrumento}

El burnout fue valorado usando la versión en español del cuestionario Maslach Burnout Inventory, (MBI), que es el que hemos utilizado para realizar este estudio, consta de 22 ítems, cada uno con una escala de respuesta de 0 (nunca) a 6 (diariamente) de los cuales 9 valoran el cansancio emocional (CE), 5 la despersonalización (DP) y 8 la realización personal (RP). El cuestionario esta validado para aplicarlo a la población Española ya que previamente se ha utilizado en otros estudios que han mostrado propiedades psicométricas satisfactorias.

Con respecto al CE, las puntuaciones de 27 o más indicarían un nivel alto, entre 19 y 26 moderado e inferiores a 19 puntos bajo.

En la subescala DP las puntuaciones superiores a 10 indicarían un nivel alto, de 6 a 9 moderado e inferior a 6 bajo. En la subescala RP los valores superiores a 40 indican una RP alta, de 34 a 39 intermedia y menor de 33 baja. En el caso de obtenerse un cansancio emocional bajo, una despersonalización baja y una alta realización personal no existe burnout. En el resto de los casos podría hablarse de burnout moderado o alto.

\section{Comité de ética y de investigación. Criterios de confidencialidad}

Todos los participantes firmaron el consentimiento informado previa explicación de en qué consistía el estudio, que debían hacer y que se podían retirar del mismo cuando quisieran. Este trabajo ha recibido el informe favorable del Comité de ética, Experimentación Animal y Bioseguridad de la Universidad Pública de Navarra (UPNA).

\section{Análisis de datos}

Para el análisis estadístico se ha usado el paquete estadístico R.

Entre las variables sociodemográficas disponibles en el cuestionario, se ha realizado una recodificación en dos de ellas para dotar a los análisis de una mayor potencia estadística. Las recodificaciones han sido las siguientes:

— En la variable "Antigüedad en el centro", las etiquetas: "20-30 años" y ">30 años" se han fusionado en la etiqueta ">20 años" ya que tan sólo un encuestado había manifestado tener una antigüedad superior a 30 años

- En la variable "Turno", dado que sólo un encuestado ha marcado la opción "Fijo de noches", se ha fusionado las etiquetas "Fijo de tardes" y "Fijo de noches" en la etiqueta "Fijo de tardes/noches". Esta fusión se ha realizado tras analizar las diferencias entre el turno fijo de noche y los otros dos turnos fijos, y observar que los valores medios en la escala de Burnout de Maslach del individuo en turno fijo de noche se acercan más a los del fijo de noche que a los de mañana.

\section{Análisis univariante}

Para cada uno de los 22 items del instrumento de medición se han realizado un análisis descriptivo basado en obtener las frecuencias, tanto absolutas como relativas de las respuestas a cada una de las opciones. Además, se ha obtenido la media y la desviación típica de cada uno de los ítems teniendo en cuenta las características de la escala (de tipo Likert de 7 puntos).

\section{Análisis bivariante}

Se han calculado las medias y las desviaciones típicas de cada uno de los ítems para cada uno de los grupos de cada una de las ocho variables sociodemográficas medidas en la encuesta. 
Junto a ello, se ha realizado para cada ítem un contraste de hipótesis de KruskalWallis. Este contraste asume en su hipótesis nula que la mediana poblacional de todos los grupos es idéntica mientras que su hipótesis alternativa asume que la mediana poblacional de alguno de los grupos es diferente al resto y por lo tanto procede de una población distinta.

Dado que el contraste de Kruskal-Wallis es no paramétrico, éste no realiza ningún tipo de suposición (normalidad, homocedasticidad...etc.) sobre los datos, por lo que resulta más adecuado de usar que el contraste de la t de Student de diferencia de medias en situaciones como las de este estudio, en las que el tamaño de muestra no permite la aplicación del Teorema Central de Límite y por ende, la t de Student.

Para aquellos ítems en los que se ha rechazado la hipótesis nula del contraste Kruskal-Wallis, se han realizado nuevos contrastes de hipótesis de Wilcoxon para comparar todos los grupos dos a dos y ver entre qué grupos existen realmente diferencias significativas. El contraste de hipótesis de Wilcoxon asume las mismas hipótesis (nula y alternativa) que el de Kruskal-Wallis, con la diferencia de que el primero sólo se puede aplicar en la comparación de la mediana de dos grupos, mientras que el segundo admite múltiples grupos.

En el contraste de hipótesis de Wilcoxon, se ha empleado la corrección del p-valor de Holm para evitar el aumento de la probabilidad de error de Tipo I que llevan asociadas las comparaciones múltiples entre grupos.

\section{Análisis multivariante}

Dada la naturaleza de la medición, las 3 subescalas deben ser analizadas de manera independiente, esto es, no es recomendable sumar las puntuaciones de los 22 ítems para formar una sola escala ya que hay relaciones inversas entre varios de ellos.

Para analizar la fiabilidad y la validez de la escala en la muestra actual, se han obtenido los coeficientes alfa de Cronbach para cada una de las 3 subescalas, así como la correlación ítem-total para cada uno de los ítems de las 3 subescalas y la diferencia de medias entre el $27 \%$ de individuos con mayor puntuación y el $27 \%$ con menor puntuación en cada uno de los ítems.

Se han obtenido modelos lineales de regresión multivariante sobre la suma de las puntuaciones para cada subescala con el objetivo de analizar la influencia de cada una de las condiciones sociodemográficas medidas en esta encuesta sobre las características medidas por el instrumento. Las variables explicativas de dichos modelos se han obtenido en base a la aplicación del algoritmo StepWise de reducción de características.

\section{RESULTADOS}

\section{Análisis univariante}

Atendiendo a las 3 subsescalas del instrumento, se observa que la media es mayor en aquellos ítems de la escala de realización, cuya relación con el Burnout es inversamente proporcional (mayor puntuación en la escala menos Burnout).

Con respecto a las otras dos subescalas, el ítem que presenta la mayor puntuación y que por lo tanto advierte de un mayor nivel de Burnout en ese sentido es la afirmación "Creo que trabajo demasiado" con una puntuación media de 3,547 puntos. Por otro lado, el ítem que notifica menor nivel de Burnout es la afirmación "Siento que realmente no me importa lo que les ocurra a mis pacientes" con una puntuación media de 1,763 puntos (figura 1). 
Figura 1. Gráfico con los intervalos de confianza (95\%) para la puntuación.

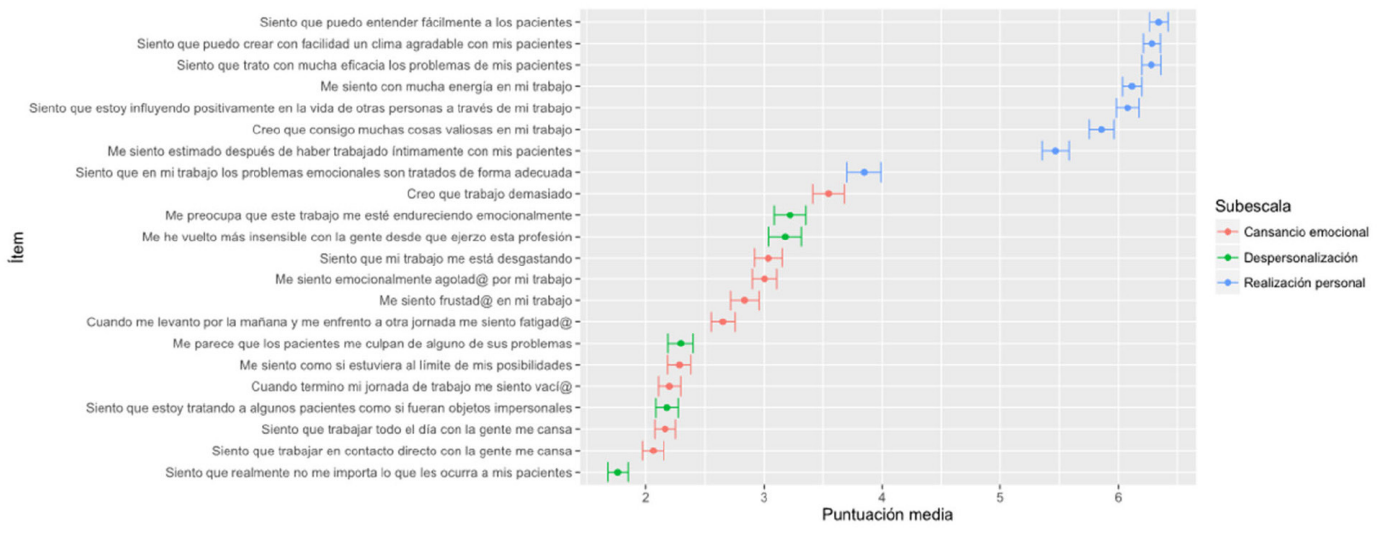

\section{Análisis bivariante}

Con respecto al ítem "Me be vuelto más insensible con la gente desde que ejerzo esta profesión" se puede apreciar que la puntuación media es dramáticamente superior entre los conductores que entre el resto de los puestos de trabajo. Estos conservadores resultados están debidos, potencialmente, al escaso tamaño de los grupos del personal de enfermería y medicina, que reduce la potencia del test y hace menos probable encontrar las diferencias existentes (tabla 1).

Tabla 1. P-valores de los tests de Wilcoxon de diferencias pareadas en la puntuación del ítem 10 y los grupos de la variable que mide el puesto de trabajo.

\begin{tabular}{l|c|c|c}
\hline & Auxiliar (TES) & Conductor (TES) & Enfermer@ \\
\hline Conductor (TES) & 0,0252 & & \\
\hline Enfermer@ & 1 & 0,1532 & \\
\hline Medic@ & 1 & 0,1469 & 1 \\
\hline
\end{tabular}

Se observa que existen diferencias en puntuación estadísticamente significativas en "Me parece que los pacientes me culpan de alguno de sus problemas", la puntuación media en este ítem de quienes llevan meses en el centro (menos de un año) es mayor que en el resto de antigüedades. Estos resultados sugieren que quienes llevan menos tiempo en el mismo centro sienten mayor carga de culpabilidad que quienes llevan más tiempo.

Existen diferencias estadísticamente significativas entre todos los grupos. Por tanto, se puede afirmar que el agotamiento emocional afecta de distinta manera al contratado fijo que al temporal. La media es considerablemente mayor entre las personas con contrato fijo que las de temporal. El mismo patrón lo presentan en el ítem "Cuando termino mi jornada de trabajo me siento vacio".

Con respecto al ítem "Siento que mi trabajo me está desgastando" se observa que la puntuación media es mucho mayor entre contratados fijos y temporales que entre funcionarios. Estos resultados afirman que el desgaste por el trabajo es diferente entre ellos y dada la media observada este desgaste es mayor entre contrataciones fijas que entre funcionarios.

En el ítem "Me he vuelto más insensible con la gente desde que ejerzo esta profesión" se observa que la puntuación media es críticamente mayor entre contratados fijos y temporales que entre el funcionariado. Por lo tanto, la insensibilidad con la gente afecta de manera distinta a funcionarios que el resto de contratados, por lo que los funcionarios presentan menos insensibilidad que el resto de contratados.

Se observa que las diferencias en la puntuación en "Creo que trabajo demasiado" son estadísticamente significativas para las comparaciones que se pueden realizar. Por lo 
que se puede afirmar que la autopercepción del exceso de trabajo es diferente en cada tipo de contrato con respecto al resto. Las medias muestrales obtenidas ayudan a trazar la dirección en la que irían estas diferencias.

En relación al agotamiento emocional en profesionales y a sentirse vacío en relación con la jornada de trabajo en trabajadores es mayor que en aquellos cuyo turno sea de 24 horas.

Respecto a "me siento frustrado en mi trabajo" se observa una mayor puntuación media entre aquellas personas con turnos de 12 horas o turno fijo de mañanas.

En relación a "Me parece que los pacientes me culpan de alguno de sus problemas" se ha observado una puntuación media particularmente alta entre las personas que tienen turno fijo de mañanas.

En el ítem "Me preocupa que este trabajo me esté endureciendo emocionalmente” se aprecia un menor promedio entre los profesionales que trabajan en zonas rurales que entre el resto. Por otro lado, respecto a "Siento que en mi trabajo los problemas emocionales son tratados de forma adecuada" el grado de acuerdo es mucho mayor, en promedio, entre los profesionales de las zonas rurales.

En los items "Me siento emocionalmente agotado con mi trabajo" y "Cuando me levanto por la mañana y me enfrento a otra jornada me siento fatigado" se observa que la puntuación media es superior entre aquellas personas que declaran un estado de salud malo o regular.

Al preguntar "Siento que trabajar todo el día con la gente me cansa", el grado de acuerdo es mayor entre la gente que reconoce tener un regular estado de salud que entre el resto. Recogiendo los p-valores de los contrastes de Wilcoxon dos a dos, se observa cómo las diferencias son estadísticamente significativas entre los individuos con regular estado de salud y aquellos con muy buen estado de salud.

Se sienten con mucha energía en su trabajo con puntuaciones medias mayores en personas que declaran un estado de salud muy bueno o excelente.

Respecto a "Siento que realmente no me importa lo que les ocurra a mis pacientes" las personas con autopercepción del estado de salud está más "polarizada" (malo o excelente) presentan una menor puntuación media que aquellas que declaran un estado de salud regular o muy bueno.

En relación al estado civil, se observa que la diferencia es estadísticamente significativa en los ítems "Siento que puedo entender fácilmente a los pacientes", "Siento que estoy tratando a algunos pacientes como si fueran objetos impersonales" y "Siento que mi trabajo me está desgastando", en los dos primeros las puntuaciones medias muestrales son mayores entre las personas casadas y en el último ítem son las personas solteras.

Tan sólo se han encontrado diferencias significativas en las puntaciones en el ítem "Siento que trabajar en contacto directo con la gente me cansa", se aprecia que la puntuación media es mayor entre aquellos que no siempre trabajan con los mismos compañeros.

\section{Análisis multivariante}

Se ha obtenido un coeficiente alpha de Cronbach de 0,91 para la subescala de cansancio emocional, 0,65 para la subescala de despersonalización y 0,71 para la subescala de realización personal. Estos valores son muy similares a los que se presentaron en la validación original de la escala, según Maslach et al. (1986), y prueban que la escala es fiable para esta muestra.

La validez convergente se ha contrastado midiendo las correlaciones ítem-total para cada ítem de las tres subescalas. Los resultados se pueden consultar en la Tabla 2. 
Tabla 2. P-valores de los tests de Wilcoxon de diferencias pareadas en la puntuación del ítem 22 y los grupos de la variable que mide la antigüedad en el centro.

\begin{tabular}{l|c|c|c|c}
\hline & $>20$ años & $\mathbf{1 ~ a ~ 5 ~ a n ̃ o s ~}$ & $\mathbf{1 0 - 2 0}$ años & 5-10 años \\
\hline 1 a 5 años & 0,8869 & & & \\
\hline $10-20$ años & 1 & 0,2846 & & \\
\hline $5-10$ años & 1 & 0,4884 & 1 & \\
\hline Meses & 0,2490 & 0,7002 & 0,0663 & 0,0941 \\
\hline
\end{tabular}

Se aprecia que todas las correlaciones son bastante altas, a excepción de la del ítem $4 \mathrm{y}$, sobretodo, la del ítem 22. Todas las correlaciones han arrojado p-valores por debajo del nivel de significación establecido para este estudio en el contraste de hipótesis de Pearson a excepción del ítem $22(\mathrm{t}=0,9498, \mathrm{~d}$. f. $=188, \mathrm{p}=0,3434)$. Se ha decidido no eliminar este ítem del cálculo de la puntuación total de la subescala, ya que supondría una modificación del instrumento y los resultados no se ven alterados en exceso, ni en la validación (alfa de Cronbach) ni en los modelos lineales obtenidos.

Por último, la validez discriminante de la escala se ha contrastado mediante el contraste $\mathrm{t}$ de Student de igualdad de medias entre el $27 \%$ de individuos con más puntuación y el $27 \%$ con menos puntuación para cada ítem. Para todos los ítems, los p-valores calculados se encuentran por debajo de 0,0001 , por lo que la validez discriminante queda probada para el instrumento en esta muestra.

Los modelos lineales obtenidos se pueden observar en la Tabla 3. Cabe destacar que para cada uno de ellos se ha comprobado que los residuos se distribuyen según una ley Normal centrada en 0 , y se ha calculado el factor de inflación de la varianza (VIF). En ningún caso el VIF se ha situado por encima del 2, por lo que se puede confirmar que no existe multicolinealidad entre las variables explicativas.

Tabla 3. Correlaciones ítem-total (excluyendo el propio ítem) de cada uno de los ítems con respecto al total de la correspondiente subescala.

\begin{tabular}{c|c|c|c|c|c}
\hline \multicolumn{2}{c|}{ Cansancio emocional } & \multicolumn{2}{c|}{ Despersonalización } & \multicolumn{2}{c}{ Realización personal } \\
\hline Ítem & Corr. ítem-total & Ítem & Corr. ítem-total & Ítem & Corr. ítem-total \\
\hline 1 & 0,7812 & 5 & 0,4313 & 4 & 0,1759 \\
\hline 2 & 0,7038 & 10 & 0,5661 & 7 & 0,2655 \\
\hline 3 & 0,6887 & 11 & 0,4706 & 9 & 0,4369 \\
\hline 6 & 0,7246 & 15 & 0,5360 & 12 & 0,5001 \\
\hline 8 & 0,7742 & 22 & 0,0691 & 17 & 0,4319 \\
\hline 13 & 0,6617 & & & 18 & 0,4607 \\
\hline 14 & 0,5294 & & & 19 & 0,5781 \\
\hline 16 & 0,6341 & & & 21 & 0,3269 \\
\hline 20 & 0,6593 & & & & \\
\hline
\end{tabular}

\section{DISCUSIÓN}

\section{Cansancio emocional}

Los factores que inciden significativamente en el cansancio emocional del profesional son el tipo de contrato, el turno, la autopercepción del estado de salud y el estado civil.

Tomando como referencia los profesionales con contrato fijo, el ser temporal disminuye de forma significativa el cansancio emocional, así como el ser funcionario (en este último caso de manera mucho más acusada). 
Tabla 4. Resumen de los modelos lineales para las tres subescalas del instrumento Burnout de Maslach.

\begin{tabular}{|c|c|c|c|c|}
\hline Variable & Etiqueta & Cansancio emocional & Despersonalización & Realización personal \\
\hline (Constante) & & $\begin{array}{l}24,722(3,015) \\
<\mathbf{0 , 0 0 0 0 1}\end{array}$ & $\begin{array}{l}11,125(1,233) \\
<0,00001\end{array}$ & $\begin{array}{l}48,590(1,951) \\
<\mathbf{0 , 0 0 0 0 1}\end{array}$ \\
\hline \multirow{2}{*}{$\begin{array}{l}\text { Tipo de contrato } \\
\text { (etiqueta de } \\
\text { referencia: fijo) }\end{array}$} & Funcionario & $\begin{array}{l}-8,43(2,851) \\
\mathbf{0 , 0 0 3 5 3}\end{array}$ & $\begin{array}{l}-5,486(1,502) \\
\mathbf{0 , 0 0 0 3 4}\end{array}$ & \\
\hline & Temporal & $\begin{array}{l}-5,596(1,798) \\
\mathbf{0 , 0 0 2 1 6}\end{array}$ & $\begin{array}{l}-0,985(0,877) \\
0,26290\end{array}$ & \\
\hline \multirow{4}{*}{$\begin{array}{l}\text { Turno } \\
\text { (etiqueta de } \\
\text { referencia: } \\
12 \text { horas) }\end{array}$} & 24 horas & $\begin{array}{l}-4,755(2,037) \\
\mathbf{0 , 0 2 0 6 6}\end{array}$ & & $\begin{array}{l}2,605(1,325) \\
\mathbf{0 , 0 5 0 8 6}\end{array}$ \\
\hline & Fijo de mañanas & $\begin{array}{l}-4,708(7,754) \\
0,54450\end{array}$ & & $\begin{array}{l}2,363(5,157) \\
0,64738\end{array}$ \\
\hline & $\begin{array}{l}\text { Fijo de tardes/ } \\
\text { noches }\end{array}$ & $\begin{array}{l}-8,757(4,677) \\
0,06282\end{array}$ & & $\begin{array}{l}8,598(3,116) \\
\mathbf{0 , 0 0 6 3 9}\end{array}$ \\
\hline & Rotatorio & $\begin{array}{l}-0,163(2,621) \\
0,95047\end{array}$ & & $\begin{array}{l}2,745(1,737) \\
0,11582\end{array}$ \\
\hline \multirow{3}{*}{$\begin{array}{l}\text { Autopercepción } \\
\text { del estado de } \\
\text { salud (etiqueta } \\
\text { de referencia: } \\
\text { excelente) }\end{array}$} & Muy buena & $\begin{array}{l}1,986(2,302) \\
0,38395\end{array}$ & \begin{tabular}{|l}
$2,378(1,226)$ \\
0,05404
\end{tabular} & $\begin{array}{l}-3,453(1,527) \\
\mathbf{0 , 0 2 4 9 8}\end{array}$ \\
\hline & Regular & $\begin{array}{l}12,833(3,181) \\
<\mathbf{0 , 0 0 0 0 1}\end{array}$ & $\begin{array}{l}5,914(1,663) \\
\mathbf{0 , 0 0 0 4 8}\end{array}$ & $\begin{array}{l}-7,367(2,107) \\
\mathbf{0 , 0 0 0 5 9}\end{array}$ \\
\hline & Mala & $\begin{array}{l}9,395(6,630) \\
0,15817\end{array}$ & $\begin{array}{l}-1,130(3,4769) \\
0,74545\end{array}$ & $\begin{array}{l}-6,125(4,420) \\
0,16757\end{array}$ \\
\hline $\begin{array}{l}\text { Estado civil } \\
\text { (etiqueta de } \\
\text { referencia: } \\
\text { casad@) }\end{array}$ & Solter@ & $\begin{array}{l}4,201(1,725) \\
\mathbf{0 , 0 1 5 8 7}\end{array}$ & & $\begin{array}{l}-2,100(1,065) \\
0,05023\end{array}$ \\
\hline \multicolumn{2}{|c|}{$\mathrm{R}^{2}$ ajustado del modelo } & 0,2022 & 0,1051 & 0,0901 \\
\hline \multicolumn{2}{|c|}{ P-valor test $\mathrm{F}$ de Fisher } & $<0,00001$ & 0,00011 & 0,001374 \\
\hline
\end{tabular}

Con respecto al turno, y tomando como referencia el cansancio emocional de los profesionales con turnos de 12 horas, el tener un turno de 24 horas supone una disminución estimada de 4,7 puntos en la escala. La disminución es mayor entre el personal fijo, y menor entre el personal rotatorio, pero en estos casos no se puede demostrar estadísticamente que la incidencia en la puntuación sea significativamente distinta de cero.

En lo que se refiere a la salud autopercibida, se observa cómo el cansancio emocional aumenta en puntuación a medida que se percibe una salud peor. Sin embargo, tan sólo se ha demostrado que este aumento en puntuación es distinto de cero para los casos en los que el encuestado declara tener un regular estado de salud.

Por último, se ha demostrado que estar soltero/a supone un aumento significativamente distinto de cero del cansancio emocional con respecto a estar casado/a. En concreto, el modelo estima que este aumento sería de 4,2 puntos en la escala.

\section{Despersonalización}

Los factores que influyen en la puntuación de despersonalización son el tipo de contrato y la salud autopercibida.

Tomando como referencia los contratados fijos, el ser funcionario supone una disminución estimada de 5,4 puntos en la escala de despersonalización, demostrándose estadísticamente que ésta es distinta de cero. No ocurre así con los contratados temporales, cuya disminución en la escala de despersonalización no puede demostrarse distinta de cero. 
En cuanto a la salud autopercibida, nuevamente se observa que la estimación es que la despersonalización aumenta a medida que empeora la autopercepción del estado de salud. Se ha podido demostrar que el aumento de despersonalización con respecto a las personas con excelente salud es distinto de cero en el caso de las personas con regular salud. Este aumento sería de 5,9 puntos en la escala.

\section{Realización personal}

Los factores que influyen en la realización personal son el turno de trabajo, la salud autopercibida y el estado civil.

Con respecto al turno, y tomando como referencia a las personas con turnos de 12 horas, se observa un aumento en la puntuación de realización para todos los turnos, en particular para el fijo de tardes/noches (el único para el que se demuestra estadísticamente que es distinto de cero) cuyo efecto se estima en un aumento de 8,6 puntos en la escala con respecto a los turnos de 12 horas.

Nuevamente, se aprecia como un peor estado de salud autopercibida resulta en peores efectos para la escala (en el sentido de mayor Burnout). Con respecto a aquellos que declaran tener una salud excelente, se estima que la realización disminuye en 3,4 puntos entre los que declaran una salud buena y en 7,4 puntos entre los que declaran una salud regular. En ambos casos, se demuestra que los efectos son significativos, esto es, distintos de cero.

Por último, se observa que el efecto del estado civil supone una disminución estimada de 2,1 puntos en la escala de realización si la persona está soltera, en comparación con si estuviera casada. Se demuestra que esta disminución es significativamente distinta de cero.

Uno de los principales problemas del Burnout, tanto internacionalmente como nacionalmente es la recuperación del enfoque psicosocial del mismo para poder visualizarlo no como una enfermedad sino como una alteración temprana de salud mental en la que destaca la importancia de la prevención ${ }^{19}$.

Por lo que el interés de este estudio radica en conocer el grado de cansancio emocional o Burnout que presentan los profesionales de urgencias extrahospitalarias y evidenciar la necesidad de tomar medidas preventivas.

Los resultados de nuestro estudio están en la línea de los publicados lo que más afecta al cansancio emocional es el tipo de contrato, disminuyendo al ser funcionarios y al tener una percepción peor de salud aumenta el Burnout.

De hecho, la Nota Técnica de Prevención (NTP) 604, herramienta de consulta en relación a la Prevención, muestra claramente que la alta tensión influye en la salud y la calidad de vida de diversas formas y ninguna de ellas beneficiosa ${ }^{20}$.

Es un punto clave a tener en cuenta por parte de la empresa, que los trabajos que impliquen riesgos especiales, tensiones físicas o mentales importantes tienen que estar definidos como tales en convenio colectivo o, en su defecto, por un acuerdo entre la empresa y los representantes de los trabajadores.

Las mayores limitaciones o sesgos de este estudio se encuentran en la mayor o menor sinceridad de los profesionales al responder al cuestionario, ya que son muchas las circunstancias que pueden afectar a la sinceridad de las respuestas.

La búsqueda de una mejor atención a los pacientes y de la prestación de mejores servicios sanitarios no puede depender sólo de aspectos técnicos, administrativos o de dotación de medios, es el factor humano lo que es irremplazable en la atención sanitaria. 


\section{DECLARACIONES}

— INTERESES COMPETITIVOS: Ningún conflicto potencial de interés fue reportado por los autores.

- CONTRIBUCIONES DE LOS AUTORES: Todos los autores contribuyeron a la concepción y el diseño del estudio, ayudaron a analizar e interpretar los resultados, revisaron el manuscrito críticamente con respecto al contenido intelectual más importante y aprobaron la versión que se publicará.

- AGRADECIMIENTOS: A todos los voluntarios que participaron en el estudio de forma gratuita, dedicando su tiempo libre para realizar un esfuerzo extra sin el cual este estudio no hubiera sido posible.

\section{REFERENCIAS BIBLIOGRÁFICAS}

1. Gil-Montes PR. El síndrome de quemarse por el trabajo (síndrome de burnout) en profesionales de enfermería. Revista Electrónica Interacao Psy. 2003; 1(1): 19-33.

2. Freudenberger HJ. Staff Burnout. J Soc Issues. 1974; 30(1): 159-65.

3. Grau Martín A, Flichentrei D, Suñer R, Prest M, Braga F. Influencia de factores personales, profesionales, transnacionales en el síndrome del Burnout en el personal sanitario hispanoamericano. Rev Esp Salud Pública 2009; 83(2): 215-30.

4. Atance JC. Aspectos epidemiológicos del síndrome de burnout en personal sanitario. Rev Esp Salud Pública 2016; 71(3): 293-303.

5. Aasa U, Brulin C, Ängquist KA, Barnekow M. 2005. Work-related psychosocial factors, worry about work conditions and health complaints among female and male ambulance personnel. Scand J Caring Sci. 19; 3: $251-8$

6. Mediano L, Fernández G. El burnout y los médicos. Un peligro desconocido. Ricardo Prats y Asociados, 2001.

7. Dickinson C, Engle P, Guise J-M, Hansen M, Jui J, Meckler G, et al. 2016. Emergency medical services responders' perceptions of the effect of stress and anxiety on patient safety in the out-of-hospital emergency care of children: a qualitative study. BMJ Open. 7; 1-6.

8. Jones MC, Wells M, Gao C, Cassidy B y Davie J. Work stress and well-being in oncology settings: a multidisplinary study of health care professionals. PsycholoOncology 2013; 22: 46-53.

9. Poghosyan L, Clarke S, Finlayson M, Aiken L. Nurse burnout and quality of care: a cross-national investigation in six countries. Res. Nurs. Health. 2010: 33; 288-298.

10. Sorour A, El-Maksoud M. Relationship between musculoskeletal disorders, job demands, and burnout among emergency nurses. Adv. Emerg. Nurs. J. 2012; 34: 272-282.

11. Iacovides A, Fountoulakis K, Moysidou C, Ierodiakonou C. Burnout in nursing staff: is there a relationship between depression and burnout?. Int. J. Psychiatry Med. 1999; 29: 421-433.

12. Moustaka E, Constantinidis T. Sources and effects of work-related stress in nursing. Health Sci. J. 2010; 4: $210-216$

13. Nichols P. 2008 Learning from error: identifying contributory causes of medication errors in an Australian hospital. Med J Just. 188: 276-9.

14. Datos SAMUR (Servicio de Asistencia Municipal de Urgencia y Rescate). Guía de Buenas Prácticas en Prevención de Riesgos Laborales SAMUR-Protección Civil. (1-53).

15. Cushman JT, Fairbanks RJ, O'Gara KG, et al. 2010. Ambulance personnel perceptions of near misses and adverse events in pediatric patients. Prehosp Emerg Care. 14; 477-84

16. Gonzalez M, Navarro P, Villar E. 2017. Psycholosocial risk and protective factors for the health and wellbeing of professionals working in emergency and non-emergency medical transport services, identified via questionnaires. Scandinavian Journal of Trauma, Resuscitation and Emergency Medicine. 25:88.

17. Martínez de la Casa Muñoz A, Del Castillo C, Magaña E, Bru I, Franco A, Segura A. Estudio sobre la prevalencia del burnout en los médicos del Área Sanitaria de Talavera de la Reina. Aten Primaria 2003; 32 (6): 343-8.

18. Estudio realizado por el Observatorio Europeo de Riesgos de la OSHA, sobre "La estimación del coste del estrés y los riesgos psicosociales relacionados con el trabajo". 
19. Juárez A, Idrova A, Camacho A, Plasencia O. 2014. Síndrome Burnout en población mexicana: una revisión sistemática. Salud Mental. 37(2): 159-67.

20. España. Ministerio de Trabajo y Asuntos Sociales. NTP 604: Riesgo psicosocial: el modelo demanda control-apoyo social (II); 2012.

| 